Annales Mathematicae Silesianae 30 (2016), 89-110

DOI: $10.1515 /$ amsil-2016-0003

\title{
FIXED POINT RESULTS SATISFYING RATIONAL TYPE CONTRACTIVE CONDITIONS IN COMPLEX VALUED METRIC SPACES
}

\author{
Poom Kumam*, Muhammad Sarwar*, Mian Bahadur Zada
}

\begin{abstract}
The aim of this manuscript is to establish fixed point results satisfying contractive conditions of rational type in the setting of complex valued metric spaces. The derived results generalize and extend some well known results in the existing literature.
\end{abstract}

\section{Introduction}

The Banach contraction principle [2] is considered to be the pioneering result of the fixed point theory, and plays an important role for solving existence problems in many branches of nonlinear analysis. This principle asserts that every contraction in a complete metric space has a unique fixed point. Inspired from the impact of this natural idea to functional analysis, several researchers have been extended and generalized this principle for different kinds of contractions in various spaces such as quasi-metric spaces, cone metric spaces, G-metric spaces and vector valued metric spaces etc.

Recently, Azam et al. [1] introduced the notion of complex valued metric space, which is one of the most attractive research topics in fixed point theory and established the result for the existence and uniqueness of a fixed point in

Received: 9.10.2015. Revised: 5.03.2016. Accepted: 15.03.2016.

(2010) Mathematics Subject Classification: 47H10, 54H25.

Key words and phrases: complex valued metric spaces, common fixed points, self-maps, Cauchy sequence, contractive conditions.

${ }^{*}$ Corresponding authors. 
complex valued metrics. Though complex valued metric spaces form a special class of cone metric space, yet this idea is intended to define rational expressions which are not meaningful in cone metric spaces and thus many results of analysis cannot be generalized to cone metric spaces. Indeed the definition of a cone metric space banks on the underlying Banach space which is not a division Ring. However, in complex valued metric spaces, we can study improvements of a host of results of analysis involving divisions. Rouzkard and Imdad [5] studied some common fixed point theorems satisfying certain rational expressions in complex valued metric spaces which generalizes the results of [1]. Sintunavarat and Kumam [8] established common fixed point theorems by replacing the constant of contractive condition to some control functions and produced results for weakly compatible mappings in the context of complex valued metric spaces. Sitthikul and Saejung [6] continue the study of fixed point theorems in complex valued metric spaces and obtained results which generalizes the results of ([8], [5]). Kumar and Hussain [4] extended the results of [6] in the context of complex valued metric spaces.

The aim of this contribution is to investigate some fixed point results using the concept of the contractive conditions of control functions in the set up of complex valued metric spaces. Actually the derived results generalizes the results of ([5], 4], 6], [8]) in complex valued metric spaces.

Now, we recollect some known definitions and results from the literature which are helpful for proving our main result.

\section{Preliminaries}

Definition 2.1 ([1]). Let $\mathbb{C}$ be the set of complex numbers and $z, w \in \mathbb{C}$. Define a partial order $\precsim$ on $\mathbb{C}$ as follows:

$$
\begin{aligned}
& z \precsim w \text { if and only if } \operatorname{Re}(z) \leq \operatorname{Re}(w) \text { and } \operatorname{Im}(z) \leq \operatorname{Im}(w) . \\
& z \prec w \text { if and only if } \operatorname{Re}(z)<\operatorname{Re}(w) \text { and } \operatorname{Im}(z)<\operatorname{Im}(w) .
\end{aligned}
$$

Note that

i) $k_{1}, k_{2} \in(0, \infty)$ and $k_{1} \leq k_{2} \Rightarrow k_{1} z \precsim k_{2} z$, for all $z \in \mathbb{C}$;

ii) $0 \precsim z \precsim w \Rightarrow|z|<|w|$, for all $z, w \in \mathbb{C}$;

iii) $z \precsim w$ and $w \prec w^{*} \Rightarrow z \prec w^{*}$, for all $z, w, w^{*} \in \mathbb{C}$.

Definition $2.2([1])$. Let $X$ be a nonempty set. Suppose that the mapping $d: X \times X \rightarrow \mathbb{C}$ satisfy the following axioms:

1) $0 \precsim d\left(z_{1}, z_{2}\right)$, for all $z_{1}, z_{2} \in X$ and $d\left(z_{1}, z_{2}\right)=0$ if and only if $z_{1}=z_{2}$; 
2) $d\left(z_{1}, z_{2}\right)=d\left(z_{2}, z_{1}\right)$, for all $z_{1}, z_{2} \in X$;

3) $d\left(z_{1}, z_{2}\right) \precsim d\left(z_{1}, z_{3}\right)+d\left(z_{3}, z_{2}\right)$, for all $z_{1}, z_{2}, z_{3} \in X$.

Then the pair $(X, d)$ is called a complex valued metric space.

ExAmple 2.3. Let $z_{1}, z_{2} \in \mathbb{C}$ and define the mapping $d: \mathbb{C} \times \mathbb{C} \rightarrow \mathbb{C}$ by

$$
d\left(z_{1}, z_{2}\right)= \begin{cases}0, & \text { if } z_{1}=z_{2} \\ i, & \text { if } z_{1} \neq z_{2} .\end{cases}
$$

Then $(\mathbb{C}, d)$ is a complex valued metric space.

Definition 2.4. [1] Let $\left\{z_{r}\right\}$ be a sequence in complex valued metric $(X, d)$ and $z \in X$. Then

i) $z$ is called the limit of $\left\{z_{r}\right\}$ if for every $w \in \mathbb{C}$, with $0 \prec w$ there is $r_{0} \in N$, such that $d\left(z_{r}, z\right) \prec w$ for all $r>r_{0}$ and we write $\lim _{r \rightarrow \infty} z_{r}=z$.

ii) $\left\{z_{r}\right\}$ is called a Cauchy sequence if for every $w \in \mathbb{C}$ with $0 \prec w$ there is $r_{0} \in N$, such that $d\left(z_{r}, z_{r+s}\right) \prec w$ for all $r>r_{0}$.

iii) $(X, d)$ is a complete complex valued metric space if every Cauchy sequence is convergent in $(X, d)$.

Lemma $2.5([1])$. Let $(X, d)$ be a complex valued metric space. Then a sequence $\left\{z_{r}\right\}$ in $X$ converges to $z$ if and only if $\left|d\left(z_{r}, z\right)\right| \rightarrow 0$ as $r \rightarrow \infty$.

Lemma $2.6([1])$. Let $(X, d)$ be a complex valued metric space. Then a sequence $\left\{z_{r}\right\}$ in $X$ is a Cauchy sequence if and only if $\left|d\left(z_{r}, z_{r+s}\right)\right| \rightarrow 0$ as $r \rightarrow \infty$, where $s \in N$.

Definition $2.7([7])$. Let $K$ and $L$ be two self-maps on a non-empty set $X$. Then

i) $z \in X$ is called a fixed point of $L$ if $L z=z$.

ii) $z \in X$ is called a coincidence point of $K$ and $L$ if $K z=L z$.

iii) $z \in X$ is called a common fixed point of $K$ and $L$ if $K z=L z=z$.

Proposition $2.8([6])$. Let $(X, d)$ be a complete complex valued metric space and $K, L: X \rightarrow X$. Let $z_{0} \in X$ and define the sequence $\left\{z_{n}\right\}$ by

$$
K z_{2 n}=z_{2 n+1} \quad \text { and } \quad L z_{2 n+1}=z_{2 n+2}, \quad n=0,1,2, \ldots
$$

Let there exists a mapping $\lambda: X \times X \rightarrow[0,1)$ satisfying

$$
\lambda(L K z, w) \leq \lambda(z, w) \quad \text { and } \quad \lambda(z, K L w) \leq \lambda(z, w) \quad \text { for all } z, w \in X
$$


Then

$$
\lambda\left(z_{2 n}, w\right) \leq \lambda\left(z_{0}, w\right) \quad \text { and } \quad \lambda\left(z, z_{2 n+1}\right) \leq \lambda\left(z, z_{1}\right)
$$

for all $z, w \in X$ and $n=0,1,2, \ldots$

Lemma $2.9([6])$. Let $(X, d)$ be a complex valued metric space and $\lambda_{1}, \lambda_{2}: X \times$ $X \rightarrow[0,1)$. If for all $z, w \in X$, the mappings $K$ and $L$ satisfy the following

$$
\begin{aligned}
d(K z, L K z) & \precsim \lambda_{1}(z, K z) d(z, K z)+\lambda_{2}(z, K z) \frac{d(z, K z) d(K z, L K z)}{1+d(z, K z)}, \\
d(K L w, L w) & \precsim \lambda_{1}(L w, w) d(L w, w)+\lambda_{2}(L w, w) \frac{d(L w, K L w) d(w, L w)}{1+d(L w, w)},
\end{aligned}
$$

then

$$
\begin{gathered}
|d(K z, L K z)| \leq \lambda_{1}(z, K z)|d(z, K z)|+\lambda_{2}(z, K z)|d(K z, L K z)|, \\
|d(K L w, L w)| \leq \lambda_{1}(L w, w)|d(L w, w)|+\lambda_{2}(L w, w)|d(L w, K L w)|,
\end{gathered}
$$

respectively.

In 2012, Sitthikul and Saejung [6] generalized the result of Rouzkard and Imdad [5] and proved the following fixed point theorem.

TheOREM $2.10([\underline{6}])$. Let $(X, d)$ be a complete complex valued metric space and $L: X \rightarrow X$ be self-map. Suppose that there exist mappings $\lambda_{1}, \lambda_{2}: X \times$ $X \rightarrow[0,1)$ such that for all $x, y \in X$

(a) $\lambda_{1}(L x, y) \leq \lambda_{1}(x, y)$ and $\lambda_{1}(x, L y) \leq \lambda_{1}(x, y)$,

$\lambda_{2}(L x, y) \leq \lambda_{2}(x, y)$ and $\lambda_{2}(x, L y) \leq \lambda_{2}(x, y)$

(b) $\lambda_{1}(x, y)+\lambda_{2}(x, y)<1$;

(c)

$$
d(L x, L y) \precsim \lambda_{1}(x, y) d(x, y)+\lambda_{2}(x, y) \frac{d(y, L y)[1+d(x, L x)]}{1+d(x, y)} .
$$

Then $L$ has a unique fixed point in $X$.

In 2012, Sitthikul and Saejung [6] generalized the result of Sintunavarat and Kumam [8] and established common fixed point result as follows:

TheOREm $2.11([6])$. Let $(X, d)$ be a complete complex valued metric space and $K, L: X \rightarrow X$ be two self-mappings. Suppose that there exist mappings $\lambda, \mu, \gamma: X \times X \rightarrow[0,1)$ such that for all $x, y \in X$ : 
(a) $\lambda(L K x, y) \leq \lambda(x, y)$ and $\lambda(x, K L y) \leq \lambda(x, y)$, $\mu(L K x, y) \leq \mu(x, y)$ and $\mu(x, K L y) \leq \mu(x, y)$ $\gamma(L K x, y) \leq \gamma(x, y)$ and $\gamma(x, K L y) \leq \gamma(x, y)$

(b) $\lambda(x, y)+\mu(x, y)+\gamma(x, y)<1$;

(c)

$$
\begin{aligned}
d(K x, L y) \precsim & \lambda(x, y) d(x, y)+\mu(x, y) \frac{d(x, K x) d(y, L y)}{1+d(x, y)} \\
& +\gamma(x, y) \frac{d(y, K x) d(x, L y)}{1+d(x, y)} .
\end{aligned}
$$

Then $K$ and $L$ have a unique common fixed point in $X$.

Kumar and Hussain [4] improved Theorem 2.12 of [6] in the following way:

ThEOREM $2.12([4])$. Let $(X, d)$ be a complete complex valued metric space and $K, L: X \rightarrow X$ be two self-mappings. Suppose that there exist mappings $\lambda, \mu, \gamma, \delta: X \times X \rightarrow[0,1)$ such that for all $x, y \in X:$

(a) $\lambda(L K x, y) \leq \lambda(x, y)$ and $\lambda(x, K L y) \leq \lambda(x, y)$,

$\mu(L K x, y) \leq \mu(x, y)$ and $\mu(x, K L y) \leq \mu(x, y)$,

$\gamma(L K x, y) \leq \gamma(x, y)$ and $\gamma(x, K L y) \leq \gamma(x, y)$

$\delta(L K x, y) \leq \delta(x, y)$ and $\delta(x, K L y) \leq \delta(x, y)$

$\eta(L K x, y) \leq \eta(x, y)$ and $\eta(x, K L y) \leq \eta(x, y)$

(b) $\lambda(x, y)+\mu(x, y)+\gamma(x, y)+2[\delta(x, y)+\eta(x, y)]<1$;

(c) $\quad d(K x, L y) \precsim \lambda(x, y) d(x, y)+\mu(x, y) \frac{d(x, K x) d(y, L y)}{1+d(x, y)}$

$$
\begin{aligned}
& +\gamma(x, y) \frac{d(y, K x) d(x, L y)}{1+d(x, y)}+\delta(x, y) \frac{d(x, K x) d(x, L y)}{1+d(x, y)} \\
& +\eta(x, y) \frac{d(y, K x) d(y, L y)}{1+d(x, y)}
\end{aligned}
$$

Then $K$ and $L$ have a unique common fixed point in $X$.

\section{Main Results}

In this section we present the main results. 
TheOrem 3.1. Let $(X, d)$ be a complete complex valued metric space and $\lambda_{i}: X \times X \rightarrow[0,1), i=1, \ldots, 8$. Suppose that $L: X \rightarrow X$ is a self-map such that for all $z, w \in X$ the following conditions are satisfied:

(i) $\lambda_{i}(L z, w) \leq \lambda_{i}(z, w)$ and $\lambda_{i}(z, L w) \leq \lambda_{i}(z, w)$;

(ii) $d(L z, L w) \precsim \lambda_{1}(z, w) d(z, w)+\lambda_{2}(z, w) d(z, L w)$

$$
\begin{aligned}
& +\lambda_{3}(z, w) d(w, L z)+\lambda_{4}(z, w) d(z, L z) \\
& +\lambda_{5}(z, w) d(w, L w)+\lambda_{6}(z, w) \frac{d(w, L w)[1+d(z, L z)]}{1+d(z, w)} \\
& +\lambda_{7}(z, w) \frac{d(z, L w)[1+d(z, L z)]}{1+d(z, w)} \\
& +\lambda_{8}(z, w) \frac{d(z, L w)[1+d(w, L z)]}{1+d(z, w) d(w, L z)}
\end{aligned}
$$

where

$$
\lambda_{1}(z, w)+\sum_{i=3}^{6} \lambda_{i}(z, w)+2\left[\lambda_{2}(z, w)+\lambda_{7}(z, w)+\lambda_{8}(z, w)\right]<1 .
$$

Then the mapping $L$ has a unique fixed point in $X$.

Proof. Let $z_{0} \in X$ and construct a sequence $\left\{z_{n}\right\}$ by the rule

$$
L z_{n}=z_{n+1}, \quad n=0,1,2, \ldots
$$

First we show that $\left\{z_{n}\right\}$ is a Cauchy sequence in $X$. For this, consider

$$
d\left(z_{n+1}, z_{n+2}\right)=d\left(L z_{n}, L z_{n+1}\right),
$$

by using condition (ii) of Theorem 3.1 with $z=z_{n}$ and $w=z_{n+1}$, we have

$$
\begin{aligned}
d\left(z_{n+1}, z_{n+2}\right) \precsim & \lambda_{1}\left(z_{n}, z_{n+1}\right) d\left(z_{n}, z_{n+1}\right)+\lambda_{2}\left(z_{n}, z_{n+1}\right) d\left(z_{n}, L z_{n+1}\right) \\
& +\lambda_{3}\left(z_{n}, z_{n+1}\right) d\left(z_{n+1}, L z_{n}\right)+\lambda_{4}\left(z_{n}, z_{n+1}\right) d\left(z_{n}, L z_{n}\right) \\
& +\lambda_{5}\left(z_{n}, z_{n+1}\right) d\left(z_{n+1}, L z_{n+1}\right) \\
& +\lambda_{6}\left(z_{n}, z_{n+1}\right) \frac{d\left(z_{n+1}, L z_{n+1}\right)\left[1+d\left(z_{n}, L z_{n}\right)\right]}{1+d\left(z_{n}, z_{n+1}\right)}
\end{aligned}
$$




$$
\begin{aligned}
& +\lambda_{7}\left(z_{n}, z_{n+1}\right) \frac{d\left(z_{n}, L z_{n+1}\right)\left[1+d\left(z_{n}, L z_{n}\right)\right]}{1+d\left(z_{n}, z_{n+1}\right)} \\
& +\lambda_{8}\left(z_{n}, z_{n+1}\right) \frac{d\left(z_{n}, L z_{n+1}\right)\left[1+d\left(z_{n+1}, L z_{n}\right)\right]}{1+d\left(z_{n}, z_{n+1}\right) d\left(z_{n+1}, L z_{n}\right)}
\end{aligned}
$$

using 3.1, we get

$$
\begin{aligned}
\left|d\left(z_{n+1}, z_{n+2}\right)\right| \leq & \lambda_{1}\left(z_{n}, z_{n+1}\right)\left|d\left(z_{n}, z_{n+1}\right)\right|+\lambda_{2}\left(z_{n}, z_{n+1}\right)\left|d\left(z_{n}, z_{n+2}\right)\right| \\
& +\lambda_{3}\left(z_{n}, z_{n+1}\right)\left|d\left(z_{n+1}, z_{n+1}\right)\right|+\lambda_{4}\left(z_{n}, z_{n+1}\right)\left|d\left(z_{n}, z_{n+1}\right)\right| \\
& +\lambda_{5}\left(z_{n}, z_{n+1}\right)\left|d\left(z_{n+1}, z_{n+2}\right)\right| \\
& +\lambda_{6}\left(z_{n}, z_{n+1}\right)\left|\frac{d\left(z_{n+1}, z_{n+2}\right)\left[1+d\left(z_{n}, z_{n+1}\right)\right]}{1+d\left(z_{n}, z_{n+1}\right)}\right| \\
& +\lambda_{7}\left(z_{n}, z_{n+1}\right)\left|\frac{d\left(z_{n}, z_{n+2}\right)\left[1+d\left(z_{n}, z_{n+1}\right)\right]}{1+d\left(z_{n}, z_{n+1}\right)}\right| \\
& +\lambda_{8}\left(z_{n}, z_{n+1}\right)\left|\frac{d\left(z_{n}, z_{n+2}\right)\left[1+d\left(z_{n+1}, z_{n+1}\right)\right]}{1+d\left(z_{n}, z_{n+1}\right) d\left(z_{n+1}, z_{n+1}\right)}\right|
\end{aligned}
$$

with the help of condition (i) of Theorem 3.1 , we get

$$
\begin{aligned}
\left|d\left(z_{n+1}, z_{n+2}\right)\right| \leq & \lambda_{1}\left(z_{0}, z_{1}\right)\left|d\left(z_{n}, z_{n+1}\right)\right|+\lambda_{2}\left(z_{0}, z_{1}\right)\left|d\left(z_{n}, z_{n+1}\right)\right| \\
& +\lambda_{2}\left(z_{0}, z_{1}\right)\left|d\left(z_{n+1}, z_{n+2}\right)\right|+\lambda_{4}\left(z_{0}, z_{1}\right)\left|d\left(z_{n}, z_{n+1}\right)\right| \\
& +\lambda_{5}\left(z_{0}, z_{1}\right)\left|d\left(z_{n+1}, z_{n+2}\right)\right|+\lambda_{6}\left(z_{0}, z_{1}\right)\left|d\left(z_{n+1}, z_{n+2}\right)\right| \\
& +\lambda_{7}\left(z_{0}, z_{1}\right)\left|d\left(z_{n}, z_{n+2}\right)\right|+\lambda_{8}\left(z_{0}, z_{1}\right)\left|d\left(z_{n}, z_{n+2}\right)\right|
\end{aligned}
$$

which implies that

$$
\begin{aligned}
\left|d\left(z_{n+1}, z_{n+2}\right)\right| \leq & \lambda_{1}\left(z_{0}, z_{1}\right)\left|d\left(z_{n}, z_{n+1}\right)\right|+\lambda_{2}\left(z_{0}, z_{1}\right)\left|d\left(z_{n}, z_{n+1}\right)\right| \\
& +\lambda_{2}\left(z_{0}, z_{1}\right)\left|d\left(z_{n+1}, z_{n+2}\right)\right|+\lambda_{4}\left(z_{0}, z_{1}\right)\left|d\left(z_{n}, z_{n+1}\right)\right| \\
& +\lambda_{5}\left(z_{0}, z_{1}\right)\left|d\left(z_{n+1}, z_{n+2}\right)\right|+\lambda_{6}\left(z_{0}, z_{1}\right)\left|d\left(z_{n+1}, z_{n+2}\right)\right| \\
& +\lambda_{7}\left(z_{0}, z_{1}\right)\left|d\left(z_{n}, z_{n+1}\right)\right|+\lambda_{7}\left(z_{0}, z_{1}\right)\left|d\left(z_{n+1}, z_{n+2}\right)\right| \\
& +\lambda_{8}\left(z_{0}, z_{1}\right)\left|d\left(z_{n}, z_{n+1}\right)\right|+\lambda_{8}\left(z_{0}, z_{1}\right)\left|d\left(z_{n+1}, z_{n+2}\right)\right| .
\end{aligned}
$$


Finally, we get

$$
\left|d\left(z_{n+1}, z_{n+2}\right)\right| \leq h\left|d\left(z_{n}, z_{n+1}\right)\right|,
$$

where

$$
h:=\frac{\lambda_{1}\left(z_{0}, z_{1}\right)+\lambda_{2}\left(z_{0}, z_{1}\right)+\lambda_{4}\left(z_{0}, z_{1}\right)+\lambda_{7}\left(z_{0}, z_{1}\right)+\lambda_{8}\left(z_{0}, z_{1}\right)}{1-\left(\lambda_{2}\left(z_{0}, z_{1}\right)+\lambda_{5}\left(z_{0}, z_{1}\right)+\lambda_{6}\left(z_{0}, z_{1}\right)+\lambda_{7}\left(z_{0}, z_{1}\right)+\lambda_{8}\left(z_{0}, z_{1}\right)\right)} .
$$

Similarly, we get

$$
\left|d\left(z_{n}, z_{n+1}\right)\right| \leq h\left|d\left(z_{n-1}, z_{n}\right)\right|
$$

Consequently,

$$
\left|d\left(z_{n+2}, z_{n+1}\right)\right| \leq h\left|d\left(z_{n+1}, z_{n}\right)\right| \leq h^{2}\left|d\left(z_{n}, z_{n-1}\right)\right| \leq \ldots \leq h^{n+1}\left|d\left(z_{1}, z_{0}\right)\right| .
$$

Now, for $m>n$, we have

$$
\begin{aligned}
d\left(z_{n}, z_{m}\right) & \precsim d\left(z_{n}, z_{n+1}\right)+d\left(z_{n+1}, z_{n+2}\right)+\ldots+d\left(z_{m-1}, z_{m}\right), \\
\left|d\left(z_{n}, z_{m}\right)\right| & \leq\left|d\left(z_{n}, z_{n+1}\right)\right|+\left|d\left(z_{n+1}, z_{n+2}\right)\right|+\ldots+\left|d\left(z_{m-1}, z_{m}\right)\right|, \\
& \leq h^{n}\left|d\left(z_{1}, z_{0}\right)\right|+h^{n+1}\left|d\left(z_{1}, z_{0}\right)\right|+\ldots+h^{m-1}\left|d\left(z_{1}, z_{0}\right)\right| \\
& \leq\left(h^{n}+h^{n+1}+\ldots+h^{m-1}\right)\left|d\left(z_{1}, z_{0}\right)\right| \\
& \leq \frac{h^{n}}{1-h}\left|d\left(z_{1}, z_{0}\right)\right| .
\end{aligned}
$$

Therefore $\lim _{n \rightarrow \infty}\left|d\left(z_{n}, z_{m}\right)\right|=0$. Hence, $\left\{z_{n}\right\}$ is a Cauchy sequence. But $X$ is complete, so there exists $t \in X$ such that $z_{n} \rightarrow t$ as $n \rightarrow \infty$.

Next, we show that $t$ is a fixed point of $L$. For this, assume that $L t \neq t$. Now

$$
d(t, L t) \precsim d\left(t, L z_{n}\right)+d\left(L z_{n}, L t\right) .
$$

By applying condition (ii) of Theorem 3.1, equation 3.2 become

$$
\begin{aligned}
d(t, L t) \precsim & d\left(t, L z_{n}\right)+\lambda_{1}\left(z_{n}, t\right) d\left(z_{n}, t\right)+\lambda_{2}\left(z_{n}, t\right) d\left(z_{n}, L t\right) \\
& +\lambda_{3}\left(z_{n}, t\right) d\left(t, L z_{n}\right)+\lambda_{4}\left(z_{n}, t\right) d\left(z_{n}, L z_{n}\right)
\end{aligned}
$$




$$
\begin{aligned}
& +\lambda_{5}\left(z_{n}, t\right) d(t, L t)+\lambda_{6}\left(z_{n}, t\right) \frac{d(t, L t)\left[1+d\left(z_{n}, L z_{n}\right)\right]}{1+d\left(z_{n}, t\right)} \\
& +\lambda_{7}\left(z_{n}, t\right) \frac{d\left(z_{n}, L t\right)\left[1+d\left(z_{n}, L z_{n}\right)\right]}{1+d\left(z_{n}, t\right)} \\
& +\lambda_{8}\left(z_{n}, t\right) \frac{d\left(z_{n}, L t\right)\left[1+d\left(t, L z_{n}\right)\right]}{1+d\left(z_{n}, t\right) d\left(t, L z_{n}\right)}
\end{aligned}
$$

with the help of equation (3.1) and condition (i) of Theorem 3.1, we can write

$$
\begin{aligned}
d(t, L t) \precsim & d\left(t, z_{n+1}\right)+\lambda_{1}\left(z_{0}, t\right) d\left(z_{n}, t\right)+\lambda_{2}\left(z_{0}, t\right) d\left(z_{n}, L t\right) \\
& +\lambda_{3}\left(z_{0}, t\right) d\left(t, z_{n+1}\right)+\lambda_{4}\left(z_{0}, t\right) d\left(z_{n}, z_{n+1}\right) \\
& +\lambda_{5}\left(z_{0}, t\right) d(t, L t)+\lambda_{6}\left(z_{0}, t\right) \frac{d(t, L t)\left[1+d\left(z_{n}, z_{n+1}\right)\right]}{1+d\left(z_{n}, t\right)} \\
& +\lambda_{7}\left(z_{0}, t\right) \frac{d\left(z_{n}, L t\right)\left[1+d\left(z_{n}, z_{n+1}\right)\right]}{1+d\left(z_{n}, t\right)} \\
& +\lambda_{8}\left(z_{0}, t\right) \frac{d\left(z_{n}, L t\right)\left[1+d\left(t, z_{n+1}\right)\right]}{1+d\left(z_{n}, t\right) d\left(t, z_{n+1}\right)} .
\end{aligned}
$$

Taking limit as $n \rightarrow \infty$, we get

$$
\begin{aligned}
d(t, L t) \precsim & \lambda_{2}\left(z_{0}, t\right) d(t, L t)+\lambda_{5}\left(z_{0}, t\right) d(t, L t)+\lambda_{6}\left(z_{0}, t\right) d(t, L t) \\
& +\lambda_{7}\left(z_{0}, t\right) d(t, L t)+\lambda_{8}\left(z_{0}, t\right) d(t, L t) .
\end{aligned}
$$

Consequently,

$$
d(t, L t) \precsim\left[\lambda_{2}\left(z_{0}, t\right)+\lambda_{5}\left(z_{0}, t\right)+\lambda_{6}\left(z_{0}, t\right)+\lambda_{7}\left(z_{0}, t\right)+\lambda_{8}\left(z_{0}, t\right)\right] d(t, L t) .
$$

The above inequality $(3.3)$ contradicts the fact that $d(t, L t) \neq 0$. Thus $L t=t$. Hence $t$ is a fixed point of $L$.

Finally, we have to show that $t$ is a unique fixed point of $L$. For this, let $t^{*} \neq t$ be another fixed point of $L$. Then on putting $z=t$ and $w=t^{*}$ in condition (ii) of Theorem 3.1, we get

$$
\begin{aligned}
d\left(t, t^{*}\right)=d\left(L t, L t^{*}\right) \precsim & \lambda_{1}\left(t, t^{*}\right) d\left(t, t^{*}\right)+\lambda_{2}\left(t, t^{*}\right) d\left(t, L t^{*}\right) \\
& +\lambda_{3}\left(t, t^{*}\right) d\left(t^{*}, L t\right)+\lambda_{4}\left(t, t^{*}\right) d(t, L t)
\end{aligned}
$$




$$
\begin{aligned}
& +\lambda_{5}\left(t, t^{*}\right) d\left(t^{*}, L t^{*}\right)+\lambda_{6}\left(t, t^{*}\right) \frac{d\left(t^{*}, L t^{*}\right)[1+d(t, L t)]}{1+d\left(t, t^{*}\right)} \\
& +\lambda_{7}\left(t, t^{*}\right) \frac{d(t, L t)[1+d(t, L t)]}{1+d\left(t, t^{*}\right)} \\
& +\lambda_{8}\left(t, t^{*}\right) \frac{d(t, L t)\left[1+d\left(t^{*}, L t\right)\right]}{1+d\left(t, t^{*}\right) d\left(t^{*}, L t\right)} .
\end{aligned}
$$

Thus

$$
\begin{aligned}
\left|d\left(t, t^{*}\right)\right| \leq & \lambda_{1}\left(t, t^{*}\right)\left|d\left(t, t^{*}\right)\right|+\lambda_{2}\left(t, t^{*}\right)\left|d\left(t, t^{*}\right)\right|+\lambda_{3}\left(t, t^{*}\right)\left|d\left(t^{*}, t\right)\right| \\
& +\lambda_{7}\left(t, t^{*}\right)\left|\frac{d\left(t, t^{*}\right)}{1+d\left(t, t^{*}\right)}\right|+\lambda_{8}\left(t, t^{*}\right)\left|\frac{d\left(t, t^{*}\right)}{1+d\left(t, t^{*}\right) d\left(t^{*}, t\right)}\right|,
\end{aligned}
$$

and consequently,

$$
\left|d\left(t, t^{*}\right)\right| \leq\left[\lambda_{1}\left(t, t^{*}\right)+\lambda_{2}\left(t, t^{*}\right)+\lambda_{3}\left(t, t^{*}\right)+\lambda_{7}\left(t, t^{*}\right)+\lambda_{8}\left(t, t^{*}\right)\right]\left|d\left(t, t^{*}\right)\right|,
$$

which is a contradiction because $\lambda_{1}\left(t, t^{*}\right)+\lambda_{2}\left(t, t^{*}\right)+\lambda_{3}\left(t, t^{*}\right)+\lambda_{7}\left(t, t^{*}\right)+$ $\lambda_{8}\left(t, t^{*}\right)<1$. Hence $t$ is a unique fixed point of $L$.

From Theorem 3.1 we can easily derive the following corollaries and the proofs of which are simple, so we omit it.

Corollary 3.2. If $\lambda_{8}=0$ and all other conditions of Theorem 3.1 are satisfied, then $L$ has a unique fixed point in $X$.

COROllary 3.3. If $\lambda_{7}=\lambda_{8}=0$ and all other conditions of Theorem 3.1 are satisfied, then $L$ has a unique fixed point in $X$.

Corollary 3.4. If $\lambda_{2}=\lambda_{3}=\lambda_{4}=\lambda_{5}=0$ and all other conditions of Theorem 3.1 are satisfied, then $L$ has a unique fixed point in $X$.

Corollary 3.5. If $\lambda_{6}=\lambda_{7}=\lambda_{8}=0$ and all other conditions of Theorem 3.1 are satisfied, then $L$ has a unique fixed point in $X$.

REMARK 3.6.

1. In Theorem 3.1 if $\lambda_{i}=0$ for $i=2,3,4,5,7,8$, we get Theorem 2.8 of [6].

2. In Theorem 3.1 if $\lambda_{i}=0$ for $i=2,3,4,5,6,7,8$, and $\lambda_{1}(\cdot)=\lambda_{1}$, we get complex valued metric version of Banach Theorem [2]. 
TheOREM 3.7. Let $(X, d)$ be a complete complex valued metric space and $\lambda_{i}: X \times X \rightarrow[0,1), i=1, \ldots, 9$. Suppose that $K, L: X \rightarrow X$ are two selfmappings such that for all $z, w \in X$ the following conditions are satisfied:

(i) $\lambda_{i}(L K z, w) \leq \lambda_{i}(z, w)$ and $\lambda_{i}(z, K L w) \leq \lambda_{i}(z, w)$;

(ii) $d(K z, L w) \precsim \lambda_{1}(z, w) d(z, w)+\lambda_{2}(z, w) \frac{d(z, K z) d(w, L w)}{1+d(z, w)}$

$$
\begin{aligned}
& +\lambda_{3}(z, w) \frac{d(w, K z) d(z, L w)}{1+d(z, w)}+\lambda_{4}(z, w) \frac{d(z, K z) d(z, L w)}{1+d(z, w)} \\
& +\lambda_{5}(z, w) \frac{d(w, K z) d(w, L w)}{1+d(z, w)} \\
& +\lambda_{6}(z, w) \frac{d(w, L w)[d(z, K z)+d(w, K z)]}{1+d(z, w)+d(K z, L w)} \\
& +\lambda_{7}(z, w) \frac{d(z, L w)[d(z, K z)+d(w, K z)]}{1+d(z, w)+d(K z, L w)} \\
& +\lambda_{8}(z, w) \frac{d(z, K z)[d(z, L w)+d(w, L w)]}{1+d(z, w)+d(K z, L w)} \\
& +\lambda_{9}(z, w) \frac{d(w, K z)[d(z, L w)+d(w, L w)]}{1+d(z, w)+d(K z, L w)}
\end{aligned}
$$

where

$$
\begin{aligned}
\sum_{i=1}^{3} \lambda_{i}(z, w)+2\left[\lambda_{4}(z, w)+\lambda_{5}(z, w)+\lambda_{7}(z, w)\right. & \left.+\lambda_{9}(z, w)\right] \\
& +3\left[\lambda_{6}(z, w)+\lambda_{8}(z, w)\right]<1 .
\end{aligned}
$$

Then $K$ and $L$ have a unique common fixed point in $X$.

Proof. Let $z_{0} \in X$ and construct a sequence $\left\{z_{n}\right\}$ by the rule

$$
K z_{2 n}=z_{2 n+1} \quad \text { and } \quad L z_{2 n+1}=z_{2 n+2}, \quad n=0,1,2, \ldots
$$

First we show that $\left\{z_{n}\right\}$ is a Cauchy sequence in $X$. For this, consider

$$
d\left(z_{2 k+1}, z_{2 k}\right)=d\left(K L z_{2 k-1}, L z_{2 k-1}\right) .
$$


By using condition (ii) of Theorem 3.7 with $z=L z_{2 k-1}$ and $w=z_{2 k-1}$, we have

$$
\begin{aligned}
& d\left(z_{2 k+1}, z_{2 k}\right) \precsim \lambda_{1}\left(L z_{2 k-1}, z_{2 k-1}\right) d\left(L z_{2 k-1}, z_{2 k-1}\right) \\
& +\lambda_{2}\left(L z_{2 k-1}, z_{2 k-1}\right) \frac{d\left(L z_{2 k-1}, K L z_{2 k-1}\right) d\left(z_{2 k-1}, L z_{2 k-1}\right)}{1+d\left(L z_{2 k-1}, z_{2 k-1}\right)} \\
& +\lambda_{3}\left(L z_{2 k-1}, z_{2 k-1}\right) \frac{d\left(z_{2 k-1}, K L z_{2 k-1}\right) d\left(L z_{2 k-1}, L z_{2 k-1}\right)}{1+d\left(L z_{2 k-1}, z_{2 k-1}\right)} \\
& +\lambda_{4}\left(L z_{2 k-1}, z_{2 k-1}\right) \frac{d\left(L z_{2 k-1}, K L z_{2 k-1}\right) d\left(L z_{2 k-1}, L z_{2 k-1}\right)}{1+d\left(L z_{2 k-1}, z_{2 k-1}\right)} \\
& +\lambda_{5}\left(L z_{2 k-1}, z_{2 k-1}\right) \frac{d\left(z_{2 k-1}, K L z_{2 k-1}\right) d\left(z_{2 k-1}, L z_{2 k-1}\right)}{1+d\left(L z_{2 k-1}, z_{2 k-1}\right)} \\
& +\lambda_{6}\left(L z_{2 k-1}, z_{2 k-1}\right) \frac{d\left(z_{2 k-1}, L z_{2 k-1}\right)\left[d\left(L z_{2 k-1}, K L z_{2 k-1}\right)+d\left(z_{2 k-1}, K L z_{2 k-1}\right)\right]}{1+d\left(L z_{2 k-1}, z_{2 k-1}\right)} \\
& +\lambda_{7}\left(L z_{2 k-1}, z_{2 k-1}\right) \frac{d\left(L z_{2 k-1}, L z_{2 k-1}\right)\left[d\left(L z_{2 k-1}, K L z_{2 k-1}\right)+d\left(z_{2 k-1}, K L z_{2 k-1}\right)\right]}{1+d\left(L z_{2 k-1}, z_{2 k-1}\right)} \\
& +\lambda_{8}\left(L z_{2 k-1}, z_{2 k-1}\right) \frac{d\left(L z_{2 k-1}, K L z_{2 k-1}\right)\left[d\left(L z_{2 k-1}, L z_{2 k-1}\right)+d\left(z_{2 k-1}, L z_{2 k-1}\right)\right]}{1+d\left(L z_{2 k-1}, z_{2 k-1}\right)} \\
& +\lambda_{9}\left(L z_{2 k-1}, z_{2 k-1}\right) \frac{d\left(z_{2 k-1}, K L z_{2 k-1}\right)\left[d\left(L z_{2 k-1}, L z_{2 k-1}\right)+d\left(z_{2 k-1}, L z_{2 k-1}\right)\right]}{1+d\left(L z_{2 k-1}, z_{2 k-1}\right)},
\end{aligned}
$$

which implies that

$$
\begin{aligned}
\left|d\left(z_{2 k+1}, z_{2 k}\right)\right| \leq & \lambda_{1}\left(L z_{2 k-1}, z_{2 k-1}\right)\left|d\left(L z_{2 k-1}, z_{2 k-1}\right)\right| \\
& +\lambda_{2}\left(L z_{2 k-1}, z_{2 k-1}\right)\left|\frac{d\left(L z_{2 k-1}, K L z_{2 k-1}\right) d\left(z_{2 k-1}, L z_{2 k-1}\right)}{1+d\left(L z_{2 k-1}, z_{2 k-1}\right)}\right| \\
& +\lambda_{5}\left(L z_{2 k-1}, z_{2 k-1}\right)\left|\frac{d\left(z_{2 k-1}, K L z_{2 k-1}\right) d\left(z_{2 k-1}, L z_{2 k-1}\right)}{1+d\left(L z_{2 k-1}, z_{2 k-1}\right)}\right| \\
& +\lambda_{6}\left(L z_{2 k-1}, z_{2 k-1}\right)\left|\frac{d\left(z_{2 k-1}, L z_{2 k-1}\right) d\left(L z_{2 k-1}, K L z_{2 k-1}\right)}{1+d\left(L z_{2 k-1}, z_{2 k-1}\right)}\right| \\
& +\lambda_{6}\left(L z_{2 k-1}, z_{2 k-1}\right)\left|\frac{d\left(z_{2 k-1}, L z_{2 k-1}\right) d\left(z_{2 k-1}, K L z_{2 k-1}\right)}{1+d\left(L z_{2 k-1}, z_{2 k-1}\right)}\right|
\end{aligned}
$$




$$
\begin{aligned}
& +\lambda_{8}\left(L z_{2 k-1}, z_{2 k-1}\right)\left|\frac{d\left(L z_{2 k-1}, K L z_{2 k-1}\right) d\left(z_{2 k-1}, L z_{2 k-1}\right)}{1+d\left(L z_{2 k-1}, z_{2 k-1}\right)}\right| \\
& +\lambda_{9}\left(L z_{2 k-1}, z_{2 k-1}\right)\left|\frac{d\left(z_{2 k-1}, K L z_{2 k-1}\right) d\left(z_{2 k-1}, L z_{2 k-1}\right)}{1+d\left(L z_{2 k-1}, z_{2 k-1}\right)}\right| .
\end{aligned}
$$

By Lemma 2.9 and equation (3.4, we get

$$
\begin{aligned}
\left|d\left(z_{2 k+1}, z_{2 k}\right)\right| \leq & \lambda_{1}\left(z_{2 k}, z_{2 k-1}\right)\left|d\left(z_{2 k}, z_{2 k-1}\right)\right|+\lambda_{2}\left(z_{2 k}, z_{2 k-1}\right)\left|d\left(z_{2 k}, z_{2 k+1}\right)\right| \\
& +\lambda_{5}\left(z_{2 k}, z_{2 k-1}\right)\left|d\left(z_{2 k-1}, z_{2 k+1}\right)\right| \\
& +\lambda_{6}\left(z_{2 k}, z_{2 k-1}\right)\left|d\left(z_{2 k}, z_{2 k+1}\right)+d\left(z_{2 k-1}, z_{2 k+1}\right)\right| \\
& +\lambda_{8}\left(z_{2 k}, z_{2 k-1}\right)\left|d\left(z_{2 k}, z_{2 k+1}\right)\right| \\
& +\lambda_{9}\left(z_{2 k}, z_{2 k-1}\right)\left|d\left(z_{2 k-1}, z_{2 k+1}\right)\right| .
\end{aligned}
$$

From Proposition 2.8 and triangular inequality, we can write

$$
\begin{aligned}
\left|d\left(z_{2 k+1}, z_{2 k}\right)\right| \leq & \lambda_{1}\left(z_{0}, z_{1}\right)\left|d\left(z_{2 k}, z_{2 k-1}\right)\right|+\lambda_{2}\left(z_{0}, z_{1}\right)\left|d\left(z_{2 k}, z_{2 k+1}\right)\right| \\
& +\lambda_{5}\left(z_{0}, z_{1}\right)\left|d\left(z_{2 k-1}, z_{2 k}\right)\right|+\lambda_{5}\left(z_{0}, z_{1}\right)\left|d\left(z_{2 k}, z_{2 k+1}\right)\right| \\
& +\lambda_{6}\left(z_{0}, z_{1}\right)\left|d\left(z_{2 k}, z_{2 k+1}\right)\right|+\lambda_{6}\left(z_{0}, z_{1}\right)\left|d\left(z_{2 k-1}, z_{2 k}\right)\right| \\
& +\lambda_{6}\left(z_{0}, z_{1}\right)\left|d\left(z_{2 k}, z_{2 k+1}\right)\right|+\lambda_{8}\left(z_{0}, z_{1}\right)\left|d\left(z_{2 k}, z_{2 k+1}\right)\right| \\
& +\lambda_{9}\left(z_{0}, z_{1}\right)\left|d\left(z_{2 k-1}, z_{2 k}\right)\right|+\lambda_{9}\left(z_{0}, z_{1}\right)\left|d\left(z_{2 k}, z_{2 k+1}\right)\right| .
\end{aligned}
$$

Finally one can get

$$
\left|d\left(z_{2 k+1}, z_{2 k}\right)\right| \leq h_{1}\left|d\left(z_{2 k}, z_{2 k-1}\right)\right|
$$

where

$$
h_{1}=\frac{\lambda_{1}\left(z_{0}, z_{1}\right)+\lambda_{5}\left(z_{0}, z_{1}\right)+\lambda_{6}\left(z_{0}, z_{1}\right)+\lambda_{9}\left(z_{0}, z_{1}\right)}{1-\left(\lambda_{2}\left(z_{0}, z_{1}\right)+\lambda_{5}\left(z_{0}, z_{1}\right)+2 \lambda_{6}\left(z_{0}, z_{1}\right)+\lambda_{8}\left(z_{0}, z_{1}\right)+\lambda_{9}\left(z_{0}, z_{1}\right)\right)} .
$$

Similarly,

$$
\left|d\left(z_{2 k-1}, z_{2 k-2}\right)\right| \leq h_{1}\left|d\left(z_{2 k-2}, z_{2 k-3}\right)\right|
$$

On the other hand, consider

$$
d\left(z_{2 k-1}, z_{2 k}\right)=d\left(K z_{2 k-2}, L K z_{2 k-2}\right) .
$$


By applying condition (ii) of Theorem 3.7 with $z=z_{2 k-2}$ and $w=K z_{2 k-2}$ to equation (3.7), we get

$$
\begin{aligned}
& d\left(z_{2 k-1}, z_{2 k}\right) \precsim \lambda_{1}\left(z_{2 k-2}, K z_{2 k-2}\right) d\left(z_{2 k-2}, K z_{2 k-2}\right) \\
&+\lambda_{2}\left(z_{2 k-2}, K z_{2 k-2}\right) \frac{d\left(z_{2 k-2}, K z_{2 k-2}\right) d\left(K z_{2 k-2}, L K z_{2 k-2}\right)}{1+d\left(z_{2 k-2}, K z_{2 k-2}\right)} \\
&+\lambda_{3}\left(z_{2 k-2}, K z_{2 k-2}\right) \frac{d\left(K z_{2 k-2}, K z_{2 k-2}\right) d\left(z_{2 k-2}, L K z_{2 k-2}\right)}{1+d\left(z_{2 k-2}, K z_{2 k-2}\right)} \\
&+\lambda_{4}\left(z_{2 k-2}, K z_{2 k-2}\right) \frac{d\left(z_{2 k-2}, K z_{2 k-2}\right) d\left(z_{2 k-2}, L K z_{2 k-2}\right)}{1+d\left(z_{2 k-2}, K z_{2 k-2}\right)} \\
&+\lambda_{5}\left(z_{2 k-2}, K z_{2 k-2}\right) \frac{d\left(K z_{2 k-2}, K z_{2 k-2}\right) d\left(K z_{2 k-2}, L K z_{2 k-2}\right)}{1+d\left(z_{2 k-2}, K z_{2 k-2}\right)} \\
&+\lambda_{6}\left(z_{2 k-2}, K z_{2 k-2}\right) \frac{d\left(K z_{2 k-2}, L K z_{2 k-2}\right)\left[d\left(z_{2 k-2}, K z_{2 k-2}\right)+d\left(K z_{2 k-2}, K z_{2 k-2}\right)\right]}{1+d\left(z_{2 k-2}, K z_{2 k-2}\right)} \\
&+\lambda_{7}\left(z_{2 k-2}, K z_{2 k-2}\right) \frac{d\left(z_{2 k-2}, L K z_{2 k-2}\right)\left[d\left(z_{2 k-2}, K z_{2 k-2}\right)+d\left(K z_{2 k-2}, K z_{2 k-2}\right)\right]}{1+d\left(z_{2 k-2}, K z_{2 k-2}\right)} \\
&+\lambda_{8}\left(z_{2 k-2}, K z_{2 k-2}\right) \frac{d\left(z_{2 k-2}, K z_{2 k-2}\right)\left[d\left(z_{2 k-2}, L K z_{2 k-2}\right)+d\left(K z_{2 k-2}, L K z_{2 k-2}\right)\right]}{1+d\left(z_{2 k-2}, K z_{2 k-2}\right)} \\
&+\lambda_{9}\left(z_{2 k-2}, K z_{2 k-2}\right) \frac{d\left(K z_{2 k-2}, K z_{2 k-2}\right)\left[d\left(z_{2 k-2}, L K z_{2 k-2}\right)+d\left(K z_{2 k-2}, L K z_{2 k-2}\right)\right]}{1+d\left(z_{2 k-2}, K z_{2 k-2}\right)} .
\end{aligned}
$$

Using equation (3.4) and Proposition 2.8, one can get

$$
\begin{aligned}
\left|d\left(z_{2 k-1}, z_{2 k}\right)\right| \leq & \lambda_{1}\left(z_{0}, z_{1}\right)\left|d\left(z_{2 k-2}, z_{2 k-1}\right)\right| \\
& +\lambda_{2}\left(z_{0}, z_{1}\right)\left|\frac{d\left(z_{2 k-2}, z_{2 k-1}\right) d\left(z_{2 k-1}, z_{2 k}\right)}{1+d\left(z_{2 k-2}, z_{2 k-1}\right)}\right| \\
& +\lambda_{4}\left(z_{0}, z_{1}\right)\left|\frac{d\left(z_{2 k-2}, z_{2 k-1}\right) d\left(z_{2 k-2}, z_{2 k}\right)}{1+d\left(z_{2 k-2}, z_{2 k-1}\right)}\right| \\
& +\lambda_{5}\left(z_{0}, z_{1}\right)\left|\frac{d\left(z_{2 k-1}, z_{2 k-1}\right) d\left(z_{2 k-1}, z_{2 k}\right)}{1+d\left(z_{2 k-2}, z_{2 k-1}\right)}\right| \\
& +\lambda_{6}\left(z_{0}, z_{1}\right)\left|\frac{d\left(z_{2 k-1}, z_{2 k}\right)\left[d\left(z_{2 k-2}, z_{2 k-1}\right)+d\left(z_{2 k-1}, z_{2 k-1}\right)\right]}{1+d\left(z_{2 k-2}, z_{2 k-1}\right)}\right|
\end{aligned}
$$




$$
\begin{aligned}
& +\lambda_{7}\left(z_{0}, z_{1}\right)\left|\frac{d\left(z_{2 k-2}, z_{2 k}\right)\left[d\left(z_{2 k-2}, z_{2 k-1}\right)+d\left(z_{2 k-1}, z_{2 k-1}\right)\right]}{1+d\left(z_{2 k-2}, z_{2 k-1}\right)}\right| \\
& +\lambda_{8}\left(z_{0}, z_{1}\right)\left|\frac{d\left(z_{2 k-2}, z_{2 k-1}\right)\left[d\left(z_{2 k-2}, z_{2 k}\right)+d\left(z_{2 k-1}, z_{2 k}\right)\right]}{1+d\left(z_{2 k-2}, z_{2 k-1}\right)}\right|
\end{aligned}
$$

with the help of Lemma 2.9, we can write

$$
\begin{aligned}
\left|d\left(z_{2 k-1}, z_{2 k}\right)\right| \leq & \lambda_{1}\left(z_{0}, z_{1}\right)\left|d\left(z_{2 k-2}, z_{2 k-1}\right)\right| \\
& +\lambda_{2}\left(z_{0}, z_{1}\right)\left|d\left(z_{2 k-1}, z_{2 k}\right)\right|+\lambda_{4}\left(z_{0}, z_{1}\right)\left|d\left(z_{2 k-2}, z_{2 k}\right)\right| \\
& +\lambda_{6}\left(z_{0}, z_{1}\right)\left|d\left(z_{2 k-1}, z_{2 k}\right)\right|+\lambda_{7}\left(z_{0}, z_{1}\right)\left|d\left(z_{2 k-2}, z_{2 k}\right)\right| \\
& +\lambda_{8}\left(z_{0}, z_{1}\right)\left|d\left(z_{2 k-2}, z_{2 k}\right)+d\left(z_{2 k-1}, z_{2 k}\right)\right|
\end{aligned}
$$

Hence

$$
\begin{aligned}
\left|d\left(z_{2 k-1}, z_{2 k}\right)\right| \leq & \lambda_{1}\left(z_{0}, z_{1}\right)\left|d\left(z_{2 k-2}, z_{2 k-1}\right)\right|+\lambda_{2}\left(z_{0}, z_{1}\right)\left|d\left(z_{2 k-1}, z_{2 k}\right)\right| \\
& +\lambda_{4}\left(z_{0}, z_{1}\right)\left|d\left(z_{2 k-2}, z_{2 k-1}\right)\right|+\lambda_{4}\left(z_{0}, z_{1}\right)\left|d\left(z_{2 k-1}, z_{2 k}\right)\right| \\
& +\lambda_{6}\left(z_{0}, z_{1}\right)\left|d\left(z_{2 k-1}, z_{2 k}\right)\right|+\lambda_{7}\left(z_{0}, z_{1}\right)\left|d\left(z_{2 k-2}, z_{2 k-1}\right)\right| \\
& +\lambda_{7}\left(z_{0}, z_{1}\right)\left|d\left(z_{2 k-1}, z_{2 k}\right)\right|+\lambda_{8}\left(z_{0}, z_{1}\right)\left|d\left(z_{2 k-2}, z_{2 k-1}\right)\right| \\
& +\lambda_{8}\left(z_{0}, z_{1}\right)\left|d\left(z_{2 k-1}, z_{2 k}\right)\right|+\lambda_{8}\left(z_{0}, z_{1}\right)\left|d\left(z_{2 k-1}, z_{2 k}\right)\right|
\end{aligned}
$$

Finally, we obtain

$$
\left|d\left(z_{2 k-1}, z_{2 k}\right)\right| \leq h_{2}\left|d\left(z_{2 k-2}, z_{2 k-1}\right)\right|
$$

where

$$
h_{2}=\frac{\lambda_{1}\left(z_{0}, z_{1}\right)+\lambda_{4}\left(z_{0}, z_{1}\right)+\lambda_{7}\left(z_{0}, z_{1}\right)+\lambda_{8}\left(z_{2 k}, z_{2 k+1}\right)}{1-\left(\lambda_{2}\left(z_{0}, z_{1}\right)+\lambda_{4}\left(z_{0}, z_{1}\right)+\lambda_{6}\left(z_{0}, z_{1}\right)+\lambda_{7}\left(z_{0}, z_{1}\right)+2 \lambda_{8}\left(z_{2 k}, z_{2 k+1}\right)\right)} .
$$

Similarly,

$$
\left|d\left(z_{2 k-3}, z_{2 k-2}\right)\right| \leq h_{2}\left|d\left(z_{2 k-4}, z_{2 k-3}\right)\right|
$$

Let $h=\max \left\{h_{1}, h_{2}\right\}$, then from equations 3.5 and 3.8 , we have

$$
\left|d\left(z_{2 k+1}, z_{2 k}\right)\right| \leq h\left|d\left(z_{2 k}, z_{2 k-1}\right)\right| \quad \text { and } \quad\left|d\left(z_{2 k-1}, z_{2 k}\right)\right| \leq h\left|d\left(z_{2 k-2}, z_{2 k-1}\right)\right| \text {. }
$$


Which implies that

$$
\left|d\left(z_{2 k+1}, z_{2 k}\right)\right| \leq h\left|d\left(z_{2 k}, z_{2 k-1}\right)\right| \leq h^{2}\left|d\left(z_{2 k-1}, z_{2 k-2}\right)\right|
$$

Consequently, we can write

$$
\left|d\left(z_{n+1}, z_{n}\right)\right| \leq h\left|d\left(z_{n}, z_{n-1}\right)\right| \leq h^{2}\left|d\left(z_{n-1}, z_{n-2}\right)\right| \leq \ldots \leq h^{n}\left|d\left(z_{1}, z_{0}\right)\right|
$$

Now, for $m>n$, we have

$$
\begin{aligned}
d\left(z_{n}, z_{m}\right) & \precsim d\left(z_{n}, z_{n+1}\right)+d\left(z_{n+1}, z_{n+2}\right)+\ldots+d\left(z_{m-1}, z_{m}\right) \\
\left|d\left(z_{n}, z_{m}\right)\right| & \leq\left|d\left(z_{n}, z_{n+1}\right)\right|+\left|d\left(z_{n+1}, z_{n+2}\right)\right|+\ldots+\left|d\left(z_{m-1}, z_{m}\right)\right| \\
& \leq h^{n}\left|d\left(z_{1}, z_{0}\right)\right|+h^{n+1}\left|d\left(z_{1}, z_{0}\right)\right|+\ldots+h^{m-1}\left|d\left(z_{1}, z_{0}\right)\right| \\
& \leq\left\{h^{n}+h^{n+1}+\ldots+h^{m-1}\right\}\left|d\left(z_{1}, z_{0}\right)\right| \\
& \leq \frac{h^{n}}{1-h}\left|d\left(z_{1}, z_{0}\right)\right| .
\end{aligned}
$$

Therefore $\lim _{n \rightarrow \infty}\left|d\left(z_{n}, z_{m}\right)\right|=0$. Hence, $\left\{z_{n}\right\}$ is a Cauchy sequence. But $X$ is complete, so there exists $t \in X$ such that $z_{n} \rightarrow t$ as $n \rightarrow \infty$.

Next, to show that $t$ is a fixed point of $K$. For this, consider

$$
d(t, K t) \precsim d\left(t, L z_{2 n+1}\right)+d\left(L z_{2 n+1}, K t\right) .
$$

Using condition (ii) of Theorem 3.7 with $z=t$ and $y=z_{2 n+1}$, we have

$$
\begin{aligned}
d(t, K t) \precsim & d\left(t, L z_{2 n+1}\right)+\lambda_{1}\left(t, z_{2 n+1}\right) d\left(t, z_{2 n+1}\right) \\
& +\lambda_{2}\left(t, z_{2 n+1}\right) \frac{d(t, K t) d\left(z_{2 n+1}, L z_{2 n+1}\right)}{1+d\left(t, z_{2 n+1}\right)} \\
& +\lambda_{3}\left(t, z_{2 n+1}\right) \frac{d\left(z_{2 n+1}, K t\right) d\left(t, L z_{2 n+1}\right)}{1+d\left(t, z_{2 n+1}\right)} \\
& +\lambda_{4}\left(t, z_{2 n+1}\right) \frac{d(t, K t) d\left(t, L z_{2 n+1}\right)}{1+d\left(t, z_{2 n+1}\right)} \\
& +\lambda_{5}\left(t, z_{2 n+1}\right) \frac{d\left(z_{2 n+1}, K t\right) d\left(z_{2 n+1}, L z_{2 n+1}\right)}{1+d\left(t, z_{2 n+1}\right)} \\
& +\lambda_{6}\left(t, z_{2 n+1}\right) \frac{d\left(z_{2 n+1}, L z_{2 n+1}\right)\left[d(t, K t)+d\left(z_{2 n+1}, K t\right)\right]}{1+d\left(t, z_{2 n+1}\right)}
\end{aligned}
$$




$$
\begin{aligned}
& +\lambda_{7}\left(t, z_{2 n+1}\right) \frac{d\left(t, L z_{2 n+1}\right)\left[d(t, K t)+d\left(z_{2 n+1}, K t\right)\right]}{1+d\left(t, z_{2 n+1}\right)} \\
& +\lambda_{8}\left(t, z_{2 n+1}\right) \frac{d(t, K t)\left[d\left(t, L z_{2 n+1}\right)+d\left(z_{2 n+1}, L z_{2 n+1}\right)\right]}{1+d\left(t, z_{2 n+1}\right)} \\
& +\lambda_{9}\left(t, z_{2 n+1}\right) \frac{d\left(z_{2 n+1}, K t\right)\left[d\left(t, L z_{2 n+1}\right)+d\left(z_{2 n+1}, L z_{2 n+1}\right)\right]}{1+d\left(t, z_{2 n+1}\right)} .
\end{aligned}
$$

Using equation (3.4) and Proposition 2.8, we get

$$
\begin{aligned}
d(t, K t) \precsim & d\left(t, z_{2 n+2}\right)+\lambda_{1}\left(t, z_{1}\right) d\left(t, z_{2 n+1}\right) \\
& +\lambda_{2}\left(t, z_{1}\right) \frac{d(t, K t) d\left(z_{2 n+1}, z_{2 n+2}\right)}{1+d\left(t, z_{2 n+1}\right)} \\
& +\lambda_{3}\left(t, z_{1}\right) \frac{d\left(z_{2 n+1}, K t\right) d\left(t, z_{2 n+2}\right)}{1+d\left(t, z_{2 n+1}\right)} \\
& +\lambda_{4}\left(t, z_{1}\right) \frac{d(t, K t) d\left(t, z_{2 n+2}\right)}{1+d\left(t, z_{2 n+1}\right)} \\
& +\lambda_{5}\left(t, z_{1}\right) \frac{d\left(z_{2 n+1}, K t\right) d\left(z_{2 n+1}, z_{2 n+2}\right)}{1+d\left(t, z_{2 n+1}\right)} \\
& +\lambda_{6}\left(t, z_{1}\right) \frac{d\left(z_{2 n+1}, z_{2 n+2}\right)\left[d(t, K t)+d\left(z_{2 n+1}, K t\right)\right]}{1+d\left(t, z_{2 n+1}\right)} \\
& +\lambda_{7}\left(t, z_{1}\right) \frac{d\left(t, z_{2 n+2}\right)\left[d(t, K t)+d\left(z_{2 n+1}, K t\right)\right]}{1+d\left(t, z_{2 n+1}\right)} \\
& +\lambda_{8}\left(t, z_{1}\right) \frac{d(t, K t)\left[d\left(t, z_{2 n+2}\right)+d\left(z_{2 n+1}, z_{2 n+2}\right)\right]}{1+d\left(t, z_{2 n+1}\right)} \\
& +\lambda_{9}\left(t, z_{1}\right) \frac{d\left(z_{2 n+1}, K t\right)\left[d\left(t, z_{2 n+2}\right)+d\left(z_{2 n+1}, z_{2 n+2}\right)\right]}{1+d\left(t, z_{2 n+1}\right)}
\end{aligned}
$$

Taking limit as $n \rightarrow \infty$, we get $d(K t, t) \precsim 0$. Thus $d(K t, t)=0$ implies that $K t=t$. Hence $t$ is a fixed point of $K$.

Analogously, using condition (ii) of Theorem 3.7 with $z=z_{2 n}$ and $w=t$ one can show that $t$ is a fixed point of $L$. Therefore $K t=L t=t$, that is $t$ is a common fixed point of $K$ and $L$.

Finally, we prove that $t$ is a unique common fixed point of $K$ and $L$. For this, suppose that $t^{*} \neq t$ be another fixed point of $K$ and $L$. Then putting 
$x=t$ and $y=t^{*}$ in condition (ii) of Theorem 3.7, we have

$$
\begin{aligned}
d\left(K t, L t^{*}\right) \precsim & \lambda_{1}\left(t, t^{*}\right) d\left(t, t^{*}\right)+\lambda_{2}\left(t, t^{*}\right) \frac{d(t, K t) d\left(t^{*}, L t^{*}\right)}{1+d\left(t, t^{*}\right)} \\
& +\lambda_{3}\left(t, t^{*}\right) \frac{d\left(t^{*}, K t\right) d\left(t, L t^{*}\right)}{1+d\left(t, t^{*}\right)}+\lambda_{4}\left(t, t^{*}\right) \frac{d(t, K t) d\left(t, L t^{*}\right)}{1+d\left(t, t^{*}\right)} \\
& +\lambda_{5}\left(t, t^{*}\right) \frac{d\left(t^{*}, K t\right) d\left(t^{*}, L t^{*}\right)}{1+d\left(t, t^{*}\right)} \\
& +\lambda_{6}\left(t, t^{*}\right) \frac{d\left(t^{*}, L t^{*}\right)\left[d(t, K t)+d\left(t^{*}, K t\right)\right]}{1+d\left(t, t^{*}\right)} \\
& +\lambda_{7}\left(t, t^{*}\right) \frac{d\left(t, L t^{*}\right)\left[d(t, K t)+d\left(t^{*}, K t\right)\right]}{1+d\left(t, t^{*}\right)} \\
& +\lambda_{8}\left(t, t^{*}\right) \frac{d(t, K t)\left[d\left(t, L t^{*}\right)+d\left(t^{*}, L t^{*}\right)\right]}{1+d\left(t, t^{*}\right)} \\
& +\lambda_{9}\left(t, t^{*}\right) \frac{d\left(t^{*}, K t\right)\left[d\left(t, L t^{*}\right)+d\left(t^{*}, L t^{*}\right)\right]}{1+d\left(t, t^{*}\right)}
\end{aligned}
$$

which implies that

$$
\begin{aligned}
\left|d\left(t, t^{*}\right)\right| \leq & \lambda_{1}\left(t, t^{*}\right)\left|d\left(t, t^{*}\right)\right|+\lambda_{3}\left(t, t^{*}\right)\left|\frac{d\left(t^{*}, t\right) d\left(t, t^{*}\right)}{1+d\left(t, t^{*}\right)}\right| \\
& +\lambda_{7}\left(t, t^{*}\right)\left|\frac{d\left(t, t^{*}\right) d\left(t^{*}, t\right)}{1+d\left(t, t^{*}\right)}\right|+\lambda_{9}\left(t, t^{*}\right)\left|\frac{d\left(t^{*}, t\right) d\left(t, t^{*}\right)}{1+d\left(t, t^{*}\right)}\right| .
\end{aligned}
$$

Consequently,

$$
\left|d\left(t, t^{*}\right)\right| \leq\left[\lambda_{1}\left(t, t^{*}\right)+\lambda_{3}\left(t, t^{*}\right)+\lambda_{7}\left(t, t^{*}\right)+\lambda_{9}\left(t, t^{*}\right)\right]\left|d\left(t^{*}, t\right)\right| .
$$

Which is a contradiction because $\lambda_{1}\left(t, t^{*}\right)+\lambda_{3}\left(t, t^{*}\right)+\lambda_{7}\left(t, t^{*}\right)+\lambda_{9}\left(t, t^{*}\right)<1$, thus $\left|d\left(t^{*}, t\right)\right|=0$ and hence $t^{*}=t$. Therefore $t$ is a unique common fixed point of $K$ and $L$.

From Theorem 3.7 we can derive the following corollaries and the proof of which is simple, so we omit it.

Corollary 3.8. If $K=L$ and all other conditions of Theorem 3.7 are satisfied, then $L$ has a unique fixed point in $X$. 
Corollary 3.9. Let $\lambda_{9}=0$ and $K, L: X \rightarrow X$ be two self-mappings satisfying all other conditions of Theorem 3.7. Then $K$ and $L$ have a unique common fixed point in $X$.

Corollary 3.10. Let $\lambda_{8}=0$ and $K, L: X \rightarrow X$ be two self-mappings satisfying all other conditions of Theorem 3.7. Then $K$ and $L$ have a unique common fixed point in $X$.

Corollary 3.11. Let $\lambda_{8}=\lambda_{9}=0$ and $K, L: X \rightarrow X$ be two selfmappings satisfying all other conditions of Theorem 3.7. Then $K$ and $L$ have a unique common fixed point in $X$.

Corollary 3.12. Let $\lambda_{7}=0$ and $K, L: X \rightarrow X$ be two self-mappings satisfying all other conditions of Theorem 3.7. Then $K$ and $L$ have a unique common fixed point in $X$.

Corollary 3.13. Let $\lambda_{7}=\lambda_{8}=\lambda_{9}=0$ and $K, L: X \rightarrow X$ be two selfmappings satisfying all other conditions of Theorem [3.7. Then $K$ and $L$ have a unique common fixed point in $X$.

Corollary 3.14. Let $\lambda_{6}=0$ and $K, L: X \rightarrow X$ be two self-mappings satisfying all other conditions of Theorem 3.7. Then $K$ and $L$ have a unique common fixed point in $X$.

Corollary 3.15. Let $\lambda_{6}=\lambda_{8}=\lambda_{9}=0$ and $K, L: X \rightarrow X$ be two selfmappings satisfying all other conditions of Theorem 3.7. Then $K$ and $L$ have a unique common fixed point in $X$.

Corollary 3.16. Let $\lambda_{2}=\lambda_{6}=\lambda_{7}=0$ and $K, L: X \rightarrow X$ be two selfmappings satisfying all other conditions of Theorem 3.7. Then $K$ and $L$ have a unique common fixed point in $X$.

Corollary 3.17. Let $\lambda_{2}=\lambda_{6}=\lambda_{7}=\lambda_{8}=\lambda_{9}=0$ and $K, L: X \rightarrow X$ be two self-mappings satisfying all other conditions of Theorem 3.7. Then $K$ and $L$ have a unique common fixed point in $X$.

Corollary 3.18. Let $\lambda_{3}=\lambda_{6}=\lambda_{7}=0$ and $K, L: X \rightarrow X$ be two selfmappings satisfying all other conditions of Theorem 3.7. Then $K$ and $L$ have a unique common fixed point in $X$.

Corollary 3.19. Let $\lambda_{3}=\lambda_{6}=\lambda_{7}=\lambda_{8}=\lambda_{9}=0$ and $K, L: X \rightarrow X$ be two self-mappings satisfying all other conditions of Theorem 3.7. Then $K$ and $L$ have a unique common fixed point in $X$. 
REMARK 3.20. In the above Theorem 3.7,

1. If $\lambda_{i}=0$ for $i=4,5,6,7,8,9$, we get Theorem 2.4 of [6].

2. If $\lambda_{i}=0$ for $i=3,4,5,6,7,8,9$, we get Corollary 2.6 of [6].

3. If $\lambda_{i}=0$ for $i=2,4,5,6,7,8,9$, we get Corollary 2.7 of [6].

4. If $\lambda_{i}=0$ for $i=6,7,8,9$, we get Theorem 2.2 of [4].

5. If $\lambda_{i}=0$ for $i=6,7,8,9$, and $K=L$, we get Corollary 2.3 of [4].

6. If $\lambda_{i}=0$ for $i=6,7,8,9$ and $\lambda_{i}(\cdot)=\lambda_{i}$, we get Theorem 10 of [3].

7. If $\lambda_{i}=0$, for $i=6,7,8,9, K=L$ and $\lambda_{i}(\cdot)=\lambda_{i}$, we get Corollary 11 of [3].

To state the next result we need the following Lemma the proof of which easily follows from Proposition 2.8 .

Lemma 3.21. Let $(X, d)$ be a complete complex valued metric space and $K, L: X \rightarrow X$. Let $z_{0} \in X$ and define the sequence $\left\{z_{n}\right\}$ by

$$
K z_{2 n}=z_{2 n+1} \quad \text { and } \quad L z_{2 n+1}=z_{2 n+2} \quad \text { for } n=0,1,2, \ldots
$$

Assume that there exists a mapping $\lambda: X \rightarrow[0,1)$ satisfying

$$
\lambda(L K z) \leq \lambda(z) \quad \text { and } \quad \lambda(K L z) \leq \lambda(z) \quad \text { for all } z \in X .
$$

Then

$$
\lambda\left(z_{2 n}\right) \leq \lambda\left(z_{0}\right) \quad \text { and } \quad \lambda\left(z_{2 n+1}\right) \leq \lambda\left(z_{1}\right) \quad \text { for all } n=0,1,2, \ldots .
$$

Proof. The proof follows from the proof of Proposition 2.8. Let $x, y \in X$ and $n=0,1,2,3, \ldots$ Then, we have

$$
\begin{aligned}
\lambda\left(x_{2 n}\right) & =\lambda\left(L K x_{2 n-2}\right) \leq \lambda\left(x_{2 n-2}\right)=\lambda\left(L K x_{2 n-4}\right) \\
& \leq \lambda\left(x_{2 n-4}\right) \leq \ldots \leq \lambda\left(x_{0}\right) .
\end{aligned}
$$

Similarly,

$$
\begin{aligned}
\lambda\left(x_{2 n+1}\right) & =\lambda\left(L K x_{2 n-1}\right) \leq \lambda\left(x_{2 n-1}\right)=\lambda\left(L K x_{2 n-3}\right) \\
& \leq \lambda\left(x_{2 n-3}\right) \leq \ldots \leq \lambda\left(x_{1}\right) .
\end{aligned}
$$

THEOREM 3.22. Let $(X, d)$ be a complete complex valued metric space and $\lambda_{i}: X \rightarrow[0,1), i=1, \ldots, 9$. Suppose that $K, L: X \rightarrow X$ are two self-mappings such that for all $z, w \in X$ the following conditions are satisfied:

(i) $\lambda_{i}(L K z) \leq \lambda_{i}(z)$ and $\lambda_{i}(K L z) \leq \lambda_{i}(z)$; 
(ii) $\quad d(K z, L w) \precsim \lambda_{1}(z) d(z, w)+\lambda_{2}(z) \frac{d(z, K z) d(w, L w)}{1+d(z, w)}$

$$
\begin{aligned}
& +\lambda_{3}(z) \frac{d(w, K z) d(z, L w)}{1+d(z, w)}+\lambda_{4}(z) \frac{d(z, K z) d(z, L w)}{1+d(z, w)} \\
& +\lambda_{5}(z) \frac{d(w, K z) d(w, L w)}{1+d(z, w)} \\
& +\lambda_{6}(z) \frac{d(w, L w)[d(z, K z)+d(w, K z)]}{1+d(z, w)} \\
& +\lambda_{7}(z) \frac{d(z, L w)[d(z, K z)+d(w, K z)]}{1+d(z, w)} \\
& +\lambda_{8}(z) \frac{d(z, K z)[d(z, L w)+d(w, L w)]}{1+d(z, w)} \\
& +\lambda_{9}(z) \frac{d(w, K z)[d(z, L w)+d(w, L w)]}{1+d(z, w)}
\end{aligned}
$$

where

$$
\lambda_{1}(z)+\lambda_{2}(z)+\lambda_{3}(z)+2\left[\lambda_{4}(z)+\lambda_{5}(z)+\lambda_{7}(z)+\lambda_{9}(z)\right]+3\left[\lambda_{6}(z)+\lambda_{8}(z)\right]<1 .
$$

Then the mappings $K$ and $L$ have a unique common fixed point in $X$.

Proof. By using Lemma 3.21 and following the same steps as in Theorem 3.7 one can easily prove the theorem.

One can deduce corollaries from Theorem 3.22 in the same way as derived from Theorem 3.7 .

REMARK 3.23. In Theorem 3.22 ,

1. If $\lambda_{i}=0$ for $i=4,5,6,7,8,9$, we get Corollary 3.2 of [6].

2. If $\lambda_{i}=0$ for $i=4,5,6,7,8,9$ and $\lambda_{1}(\cdot)=\lambda_{1}, \lambda_{2}(\cdot)=\lambda_{2}, \lambda_{3}()=.\lambda_{3}$, we get Theorem 2.1 of [5].

3. If $\lambda_{i}=0$ for $i=3,4,5,6,7,8,9$, we get Theorem 3.1 of 8 .

4. If $\lambda_{i}=0$ for $i=3,4,5,6,7,8,9$ and $\lambda_{1}(\cdot)=\lambda_{1}, \lambda_{2}(\cdot)=\lambda_{2}$, we get Theorem 4 of [1].

Competing Interest. The authors declare that they have no competing interests. 
Author's contributions. All authors read and approved the final version.

Acknowledgments. The authors are grateful for the reviewers for the careful reading of the paper and for the suggestions which improved the quality of this work.

\title{
References
}

[1] Azam A., Fisher B., Khan M., Common fixed point theorems in complex valued metric spaces, Numer. Funct. Anal. Optim. 32 (2011), no. 3, 243-253.

[2] Banach S., Sur les opérations dans les ensembles abstraits et leurs applications aux equations integrales, Fund. Math. 3 (1922), 133-181.

[3] Klin-eam C., Suanoom C., Some common fixed point theorems for generalized contractive type mappings on complex valued metric spaces, Abstr. Appl. Anal. 2013, Art. ID 604215, 6 pp.

[4] Kumar T.S., Hussain R.J., Common fixed point theorems in complex valued metric spaces, Int. J. Innov. Res. Sci. Engrg. 2 (2014), no. 14, 834-838.

[5] Rouzkard F., Imdad M., Some common fixed point theorems on complex valued metric spaces, Comput. Math. Appl. 64 (2012), no. 6, 1866-1874.

[6] Sitthikul K., Saejung S., Some fixed point theorems in complex valued metric space, Fixed Point Theory Appl. 2012, 189, 11 pp.

[7] Sintunavarat W., Kumam P., Common fixed point theorem for a pair of weakly compatible mappings in fuzzy metric space, J. Appl. Math. 2011, Art. ID 637958, 14 pp.

[8] Sintunavarat W., Kumam P., Generalized common fixed point theorems in complex valued metric spaces and applications, J. Inequal. Appl. 2012, 84, 12 pp.

\author{
Poom Kumam \\ Department of Mathematics \\ Faculty of Science \\ 126 Pracha Uthit Road \\ Bang Mod, Thung Khru \\ BANGKOK 10140 \\ THAILAND \\ e-mail: poom.kum@kmutt.ac.th \\ Muhammad Sarwar, Mian Bahadur Zada \\ Department of Mathematics \\ UNIVERSITY OF MALAKAND \\ ChaKdara Dir(L) \\ PAKISTAN \\ e-mail: sarwarswati@gmail.com \\ e-mail: mbz.math@gmail.com
}

King Mongkut's University of Technology Thonburi (KMUTT) 